\title{
Fault Prognosis of Polymerase Chain Reaction Thermal Cycler Using Temperature Analysis
}

\author{
Jong-Dae Kim, ${ }^{1,2}$ So-Yeon Lee, ${ }^{1,2}$ Yu-Seop Kim, ${ }^{1,2}$ \\ Hye-Jeong Song, ${ }^{1,2}$ and Chan-Young Park ${ }^{1,2^{*}}$ \\ ${ }^{1}$ Department of Convergence Software, Hallym University, \\ 1, Hallimdaehak-gil, ChunCheon-si, Gangwon-do 24252, Republic of Korea \\ ${ }^{2}$ Bio-IT Research Center, Hallym University, \\ 1, Hallimdaehak-gil, ChunCheon-si, Gangwon-do 24252, Republic of Korea
}

(Received May 31, 2018; accepted October 18,2018)

Keywords: fault prognosis, remaining useful life, prognostics and health management, PCR thermal cycler

In this paper, we propose an abnormal detection and defect prognostic method for a polymerase chain reaction (PCR) thermal cycler. PCR is a very important technology that is used to manipulate DNA or RNA in molecular biology. Thus, predicting the aging and residual useful life of the PCR device is essential to minimize the occurrence of catastrophic failure of the device. The proposed method predicts the aging and residual useful life of the PCR thermocycler by analyzing the change in the temperature regulation rate for each of the heating and cooling sections in the temperature profile. In this study, we analyzed three PCR thermal cyclers using the proposed method and showed that the proposed method can be used to measure aging.

\section{Introduction}

Prognostics and health management (PHM) is a predictive and effective health management technology for diagnostic purposes ${ }^{(1)}$ and remaining useful life (RUL), wherein the status of equipment and mechanical systems is monitored using sensors and signs of failure are detected. ${ }^{(2)}$ Proactive diagnostics and system state management can predict the remaining lifetime by making use of the ability to assess the current state and capture the gradual degradation of the asset. $^{(3)}$ It is essential to predict machine failure and minimize maintenance costs, downtime, and safety risks. The RUL is the key to the state-based maintenance and prognosis of devices. ${ }^{(4)}$ With accurate RUL predictions, an effective solution to the management of the asset can be prepared in advance.

Methods of predicting device defects are divided into three categories: physical-model-based defect prognosis, data-based failure prognosis, and experience-based defect prognosis from a methodological point of view. The data-based approach generates fault models directly from the sensor's collected data. ${ }^{(5,6)}$ In this paper, a failure model is created on the basis of real-time

*Corresponding author: e-mail: cypark@hallym.ac.kr https://doi.org/10.18494/SAM.2019.2098 
data of temperature and time accumulated while operating a polymerase chain reaction (PCR) device. The data collected in this approach is analyzed using various statistical data pattern recognition systems, and is used to detect changes in variable data on time and predict failures. ${ }^{(2)}$

PCR is a molecular biologic technology used to diagnose and analyze diseases by replicating or amplifying specific DNA regions. ${ }^{(7)}$ The PCR process proceeds by setting the temperature value and time in the PCR thermal cycler. A thermal cycler is the most commonly used machine to amplify DNA fragments by PCR. ${ }^{(8)}$ The PCR thermal cycler performs a thermocycler function that raises or lowers the temperature to individual preprogrammed steps. The performance of the PCR thermal cycler depends on the rate of temperature regulation. Therefore, by measuring PCR thermal cycler, it is possible to predict aging or failure of the device. Since the PCR chip generally operates at about $10{ }^{\circ} \mathrm{C}$ per second and controls the temperature within $0.5{ }^{\circ} \mathrm{C}$, the processing period of the ControlProc should be much smaller than $1 / 20(50 \mathrm{~ms}) .{ }^{(9)}$ If it takes more than one second to control the temperature, the device is considered to behave abnormally. Diagnosing the abnormal behavior of the device and predicting the remaining lifetime can increase asset reliability and reduce maintenance costs.

In this paper, we predict the failure of the PCR device and estimate the RUL using the condition-monitoring data of the PCR device. Heating and cooling rates are expected to decrease the longer the device is used. Therefore, heating and cooling times will increase. In the temperature profile, the change in the temperature regulation rate was analyzed for each of the heating and cooling sections. The PCR device may inadvertently be shut down owing to external or internal factors while operating in accordance with the set temperature protocol. In this case, there is a gap in the state-monitoring data, and the time for controlling the PCR thermal cycle temperature is overly long, making it difficult to analyze the change in temperature control speed. To solve this problem, the autoregressive model was first used to filter out signals that are not fault related. ${ }^{(10)}$ The filtered signals use the exponentially weighted moving average (EWMA) filter to remove the measurement noise and find an optimal estimate. The failure threshold for predicting the failure of the PCR instrument senses the onset of aging and estimates the RUL using an appropriate data-based method. On the basis of this approach, we predict new defects in the PCR thermal cycler.

In Sect. 2, the method of predicting the aging of the PCR thermal cycler is described, and in Sect. 3, we give the experimental results obtained using the temperature profile of the PCR device to demonstrate the efficiency of the proposed method.

\section{Materials and Methods}

Table 1 shows an example of a general PCR protocol used in the PCR device. Detecting the onset of performance degradation is an effective way of predicting defects rather than accurately predicting the RUL when the PCR device is healthy. The purpose of this experiment is not to confirm the execution result of the PCR device, but to measure the aging of the PCR device. Therefore, the PCR protocol used in the experiment is simpler than the existing PCR protocol. As per the simplified protocol, the PCR thermal cycler is repeatedly heated and cooled to reach a temperature in the range from 8 to $95^{\circ} \mathrm{C}$. 
Table 1

Example of general PCR protocol.

\begin{tabular}{lcc}
\hline Label & Temperature $\left({ }^{\circ} \mathrm{C}\right)$ & Duration $(\mathrm{s})$ \\
\hline 1 & 50 & 10 \\
2 & 95 & 10 \\
3 & 50 & 10 \\
4 & 72 & 10 \\
5 & 95 & 10 \\
6 & 50 & 10 \\
\hline
\end{tabular}

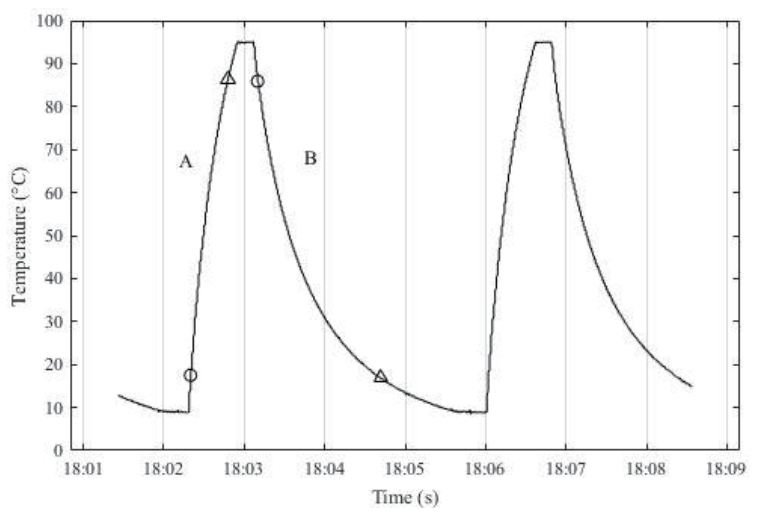

Fig. 1. Example of general protocol.

Figure 1 shows the temperature profile obtained by performing the simplified protocol. Since the PCR thermal cycler maintains the target temperature for $10 \mathrm{~s}$, it is difficult to accurately specify the starting points of the rising and falling sections. Therefore, 10 to $90 \%$ of the target temperature was designated as the ascending and descending sections. ${ }^{(11,12)}$ In Fig. 1, 10 and $90 \%$ of the target temperature are marked with ' $O$ ' and ' $\Delta$ ' in each temperature cycle. A is the heating section and B is the cooling section, and heating and cooling times are calculated in each section.

Noise in the PCR temperature-monitoring data can be caused by several factors. In particular, noise is mostly generated by sensor inaccuracy, erroneous measurements, and unpredictable noise. Unpredictable noise almost certainly originates from a faulty sensor installed in the unit. The PCR instrument data includes temperature and time values. If the PCR device is stopped during operation in accordance with the set protocol, there will be a blank in the temperature and time values. In this case, the program that monitors each cycle may calculate the heating and cooling times as being overly long. Hence, it is important to remove noise to extract time data for accurate temperature control.

Therefore, the rising and falling periods are separated in the temperature profile, noise is removed using the EWMA filter for each interval, and the temperature control time is measured. As the equipment ages, the heating and cooling rates will decrease and the time taken to control the temperature will increase. Therefore, since recent measurements are closer to present measurements than older measurements, a large weight is given to recent measurements, and a small weight is given to older measurements.

$$
\bar{x}_{k}=\alpha \bar{x}_{k-1}+(1-\alpha) x_{k}
$$

Here, $\bar{x}_{k}$ is the average of $n$ data from $(k-n+1)$ th data to $k$ th data and $\alpha$ is a constant with $0<\alpha<1$. The noise of data was removed using the EWMA filter and autoregressive model, and the higher data was returned to the estimated value at a higher rate. In the function, the $\alpha$ value for adjusting the weight is set to 0.3 . As a result, the noise of the measurement data was reduced and the trend of the altitude change was good. 
For each cycle, the data of the cooling and heating sections were analyzed using the EWMA filter and autoregressive model. In Figs. 2 and 3, the blue line indicates the data analyzed by the proposed method and the red line the actual measured data. The $x$-axis represents the number of samples obtained by the proposed method and the $y$-axis the time at which the target temperature is reached. Since the EWMA filter is also referred to as a low-pass filter (LPF), it is indicated as LPF in the figure legend. As shown in the figure, the noise of the data was reduced and the trend of the altitude change was good.

\section{Results}

After analyzing the data using the proposed method, the moving average of each interval is calculated. Figure 4 shows the moving average of the heating section. Figure 5 shows the moving average of the cooling section. As a result, the time required for heating after the PCR device has performed 3500 temperature cycles gradually increases. When the results

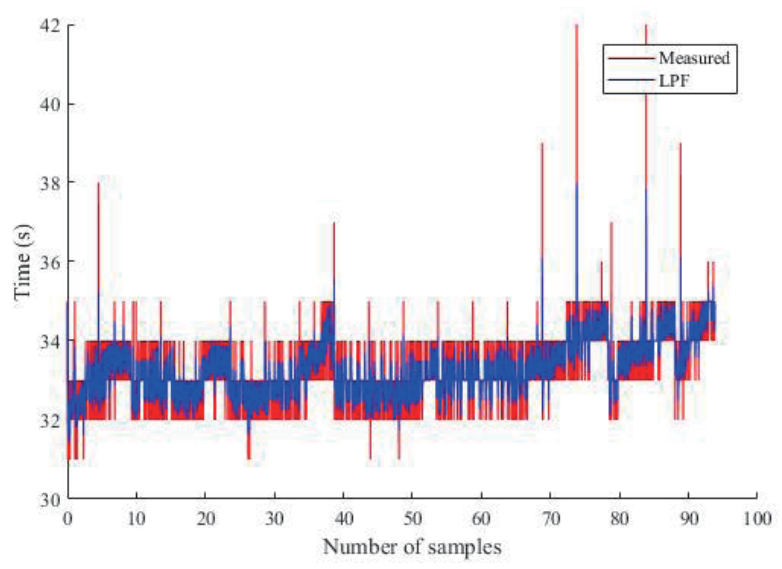

Fig. 2. (Color online) Data of measured and processed heating sections.

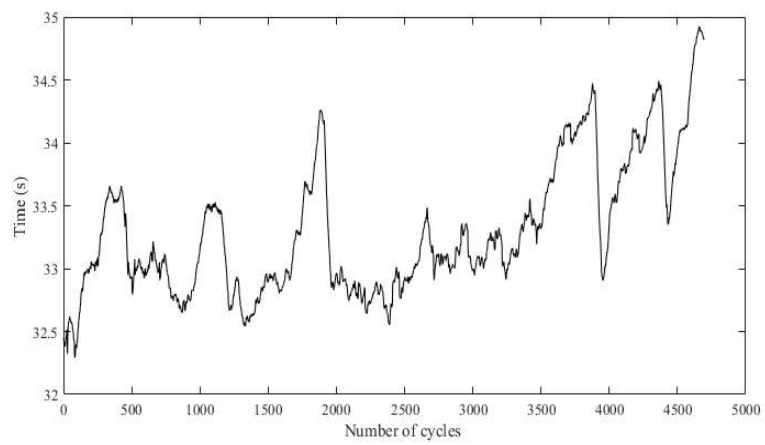

Fig. 4. Moving average of heating section.

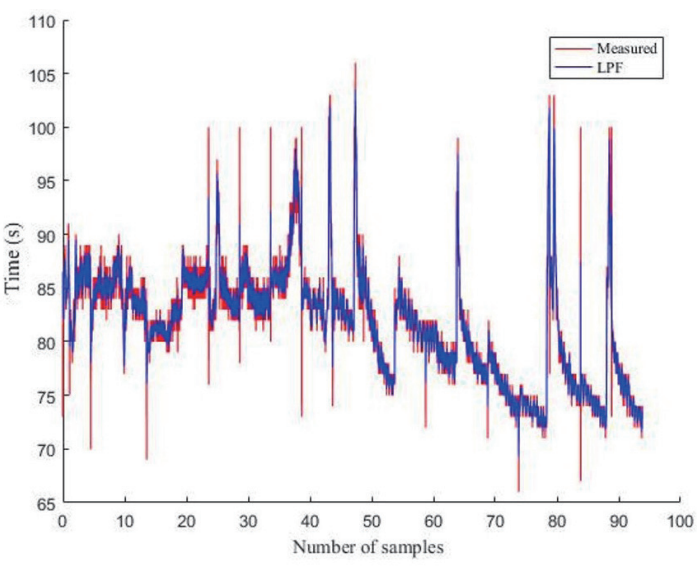

Fig. 3. (Color online) Data of measured and processed cooling sections.

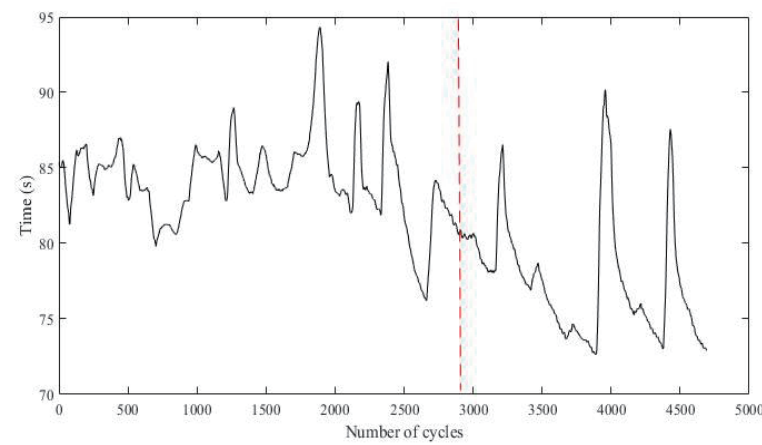

Fig. 5. (Color online) Moving average of cooling section. 
are analyzed in Fig. 4, a failure prognosis is obtained after the 3500 temperature cycles. The heating speed is found to decrease. However, it can be confirmed that the target temperature of $95{ }^{\circ} \mathrm{C}$ is reached and the amplification is normally performed. On the other hand, when the results are analyzed in Fig. 5, the cooling time is observed to gradually decrease. At this time, the target cooling temperature may not reach $8{ }^{\circ} \mathrm{C}$, and the time may seem to be shortened because the device stops in the middle of cooling. When analysis is not possible using only data, it is necessary to have a supervisor determine the fault on the basis of experience.

In the cooling section, the 2960th cycle does not reach the target temperature of $8{ }^{\circ} \mathrm{C}$. Therefore, the 2960th cycle can be considered a fault cycle. In Fig. 5, the vertical line indicates the start of failure.

\section{Conclusions}

In a PCR device, the thermal cycler plays an important role in heating and cooling of the chamber. Therefore, the detection of abnormalities and failure prediction of the thermal cycler is essential for the reliable operation of the PCR device. In order to predict the aging of the PCR thermal cycler, we analyzed the temperature control rate of the PCR thermal cycler over time. In the temperature profile, the heating and cooling sections were separated and averaged, and the variation in the temperature regulation speed was tracked in each section.

As a result of the experiment, we found that the proposed approach can give advance warning regarding the condition of the PCR thermal cycler. If the PCR device performs the setting temperature protocol only about 2960 times, it is considered that the device is beginning to age. If the PCR device is used five times a day, the device will operate normally for about 1 year and 8 months. If the PCR device is checked after 1 year and 6 months unexpected errors can be avoided. Therefore, the proposed method can successfully detect abnormal phenomena of the PCR device, schedule the maintenance, and avoid unexpected errors.

In this study, we collected data through sensors attached to the PCR thermal cycler. However, data on external covariances as well as condition monitoring data are needed. If the ambient temperature is abnormally high, it may affect the components of the PCR device and consequently speed up or slow down the heating and cooling of the camber. Therefore, data on external covariances are also needed to measure the failure rate and correct RUL. If you can manage PCR monitoring data in the cloud in combination with Internet of Things (IoT) technology in the future, you can manage your data anywhere.

\section{Acknowledgments}

This research was supported by The Leading Human Resource Training Program of Regional Neo Industry through the National Research Foundation of Korea (NRF) funded by the Ministry of Science, ICT and Future Planning (NRF-2016H1D5A1909654). 


\section{References}

1 D. Kwon, M. R. Hodkiewicz, J. Fan, T. Shibutani, and M. G. Pecht: IEEE Access 4 (2016) 3659. https://doi. org/10.1109/ACCESS.2016.2587754

2 X.-S. Si, W. Wang, C.-H. Hu, and D.-H. Zhou: Eur. J. Oper. Res. 213 (2011) 1. https://doi.org/10.1016/ j.ejor.2010.11.018

3 D. Galar, A. Thaduri, M. Catelani, and L. Ciani: Measurement 67 (2015) 137. https://doi.org/10.1016/ j.measurement.2015.01.015

4 Z. Huang, Z. Xu, W. Wang, and Y. Sun: IEEE Trans. Reliab. 64 (2015) 687. https://doi.org/10.1109/ TR.2015.2403433

5 T. Escobet, J. Quevedo, and V. Puig: 2012 Int. Joint Conf. Neural Networks (IJCNN) (2012) 1. https://doi. org/10.1109/IJCNN.2012.6252688

6 D. Solomatine, L. M. See, and R. Abrahart: Data-Driven Modelling: Concepts, Approaches and Experiences, in Practical Hydroinformatics (Springer, Heidelberg, 2009) p. 17. https://doi.org/10.1007/978-3-540-79881-1 2

7 K. Mullis, F. Faloona, S. Scharf, R. Saiki, G. Horn, and H. Erlich: Cold Spring Harbor Symp. Quant. Biol. 51 (1986) 263. https://doi.org/ 10.1101/SQB.1986.051.01.032

8 G. C. Saunders, J. Dukes, H. C. Parkes, and J. H. Cornett: Clin. Chem. 47 (2001) 47.

9 J.-H. Kim, J.-D. Kim, H.-J. Lim, and J. Kim: JKIIT 9 (2011) 7.

10 X. Jin, Y. Sun, Z. Que, Y. Wang, and T. W. Chow: IEEE Trans. Instrum. Meas. 65 (2016) 2046.

11 W. C. Elmore: J. Appl. Phys. 19 (1948) 55.

12 M.-S. Lee, C.-Y. Park, Y.-S. Kim, H.-J. Song, and J.-D. Kim: Adv. Sci. Technol. Lett. 143 (2017) 113.

\section{About the Authors}

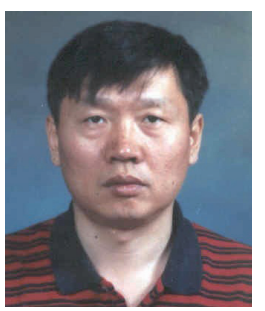

Jong-Dae Kim received his M.S. and Ph.D. degrees in electrical engineering from the Korea Advanced Institute of Science and Technology, Seoul, Korea, in 1984 and 1990, respectively. He worked for Samsung Electronics from 1988 to 2000 as an electrical engineer. He is currently a professor in the Department of Convergence Software, Hallym University. His recent interests focus on biomedical systems and bioinformatics.

(kimjd@hallym.ac.kr)

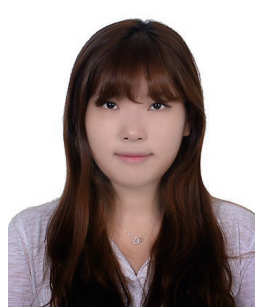

So-Yoen Lee graduated from Hallym University in 2017 and now attends the graduate school of the Department of Convergence Software, Hallym University. She is studying embedded systems and cloud computing at the Bio-IT lab. Her recent interests are convergence of biomedical equipment and IoT technology. (ye0721on@hallym.ac.kr)

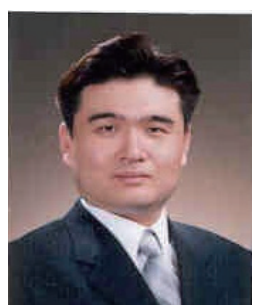

Yu-Seop Kim received his Ph.D. degree in computer engineering from Seoul National University in 2000. He is currently a professor in the Department of Convergence Software at Hallym University, South Korea. His research interests are in the areas of bioinformatics, computational intelligence, and natural language processing. (yskim01@hallym.ac.kr) 


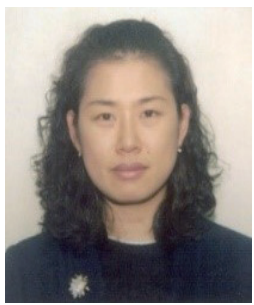

Hye-Jeong Song received her Ph.D. degree in computer engineering from Hallym University in 2003. She is currently a professor in the Department of Convergence Software at Hallym University. Her recent research has been in the areas of biomedical systems and bioinformatics. (hjsong@hallym.ac.kr)

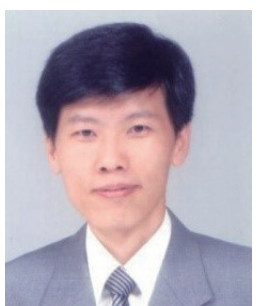

Chan-Young Park received his B.S. degree from Seoul National University in 1987 and his M.S. and Ph.D. degrees from the Korea Advanced Institute of Science and Technology in 1989 and 1995, respectively. From 1991 to 1999, he worked for Samsung Electronics. He is currently a professor in the Department of Convergence Software at Hallym University. His research interests are in the areas of Bio-IT convergence, intelligent transportation systems, and sensor networks. (cypark@hallym.ac.kr) 